\title{
BMJ Open Neurophysiological changes of brain and spinal cord in individuals with patellofemoral pain: a systematic review and meta-analysis protocol
}

\author{
Jing Nong Liang (D) , Savanna Budge, Austin Madriaga, Kara Meske, \\ Derrick Nguyenton, Kai-Yu Ho (D)
}

To cite: Liang JN,

Budge S, Madriaga A, et al. Neurophysiological changes of brain and spinal cord in individuals with patellofemoral pain: a systematic review and metaanalysis protocol. BMJ Open 2021;11:e049882. doi:10.1136/ bmjopen-2021-049882

- Prepublication history for this paper is available online. To view these files, please visit the journal online (http://dx.doi. org/10.1136/bmjopen-2021 049882).

Received 03 February 2021 Accepted 12 July 2021

\section{Check for updates}

(C) Author(s) (or their employer(s)) 2021. Re-use permitted under CC BY-NC. No commercial re-use. See rights and permissions. Published by BMJ.

Department of Physical Therapy, University of Nevada, Las Vegas, Las Vegas, Nevada, USA

Correspondence to

Dr Kai-Yu Ho;

kaiyu.ho@unlv.edu

\section{ABSTRACT}

Introduction Reduced neuromuscular control due to altered neurophysiological functions of the central nervous system has been suggested to cause movement deficits in individuals with patellofemoral pain (PFP). However, the underlying neurophysiological measures of brain and spinal cord in this population remain to be poorly understood. The purpose of this systematic review is to evaluate the evidence for altered cortical and spinal cord functions in individuals with PFP.

Methods and analysis The protocol for conducting the review was prepared using the Preferred Reporting Items for Systematic Review and Meta-Analysis Protocols guidelines. We will systematically search the literature that examines cortical and spinal cord functions in individuals with PFP, aged 18-45 years. The studies for cross-sectional, prospective, longitudinal, case-control and randomised control trial designs will be included from the following databases: PubMed (MEDLINE), EMBASE and Web of Science. Only studies published in English prior to 1 February 2021 will be included. The risk of bias and quality assessment will be performed using National Institutes of Health's Quality Assessment Tool for Observational Cohort and Cross-Sectional Studies. We will conduct meta-analysis of the data where appropriate. Narrative synthesis will be taken if a meta-analysis is not possible.

Ethics and dissemination This is a systematic review from the existing literature and does not require ethical approval. The results of this study will be published in a peer-reviewed journal in the field of rehabilitation medicine, sports/orthopaedic medicine or neurology, regardless of the outcome.

PROSPERO registration number CRD42020212128.

\section{INTRODUCTION}

Patellofemoral pain (PFP) is prevalent throughout the lifespan, affecting not only the general population but also specific populations such as adolescents, highly active individuals and the military, with an incidence rate of $9 \%-15 \% .^{1}$ Furthermore, women are 2.23 times more likely to experience PFP than men, with a prevalence of $12 \%-13 \%$ in those
Strengths and limitations of this study

- This is the first systematic review of functional changes in brain and spinal cord from chronic patellofemoral pain

- This protocol was written using the Preferred Reporting Items for Systematic Review and MetaAnalysis Protocols guidelines.

- Articles will be assessed for risk of bias and methodological quality by two independent researchers.

- Where appropriate, meta-analysis will be performed.

- One limitation is that studies not published in English will be excluded; thus, the review results may not represent all literature in this topic.

aged 18-35 years. $^{2}$ One hallmark symptom of PFP is pain around or behind the patella, which is often exacerbated by loading of the patellofemoral joint in a flexed knee position. ${ }^{34}$

Individuals with PFP often exhibit difficulty performing weight-bearing tasks such as negotiating stairs, squatting and running. ${ }^{3}$ For example, an increase in dynamic knee valgus is a common movement deficit observed during those functional movements in this population. ${ }^{56}$ This atypical pattern is the result of excessive hip adduction and internal rotation, which causes excessive loading to the lateral aspect of patella and PFP. ${ }^{5}$ In addition, increased hip adduction during functional activities has been found to be a contributing factor of a higher level of pain and dysfunction in women with PFP. $^{7}$ As weakness of hip musculature (ie, hip abductors and hip external rotators) is believed to attribute to excessive knee valgus during weight-bearing activities ${ }^{5}$ addressing hip strength deficits is a commonly theorised treatment for such faulty movements. ${ }^{8}$ However, while hip muscle strengthening programmes have been shown to reduce pain and hip weakness deficits, the evidence supporting hip muscle strengthening 
on improving dynamic knee valgus during functional activities is limited..$^{8-10}$ A recent systematic review with meta-analysis further suggests that hip muscle weakness is not a risk factor of developing future PFP in adults. ${ }^{11}$

In fact, as neuromuscular control is essential while performing functional movements, it has been found that a gait retraining protocol effectively corrects the frontal plane movement deficits during running in individuals with PFP, while a hip muscle strengthening programme alone does not. ${ }^{9}$ These gait retraining protocols often incorporate motor learning principles, such as faded feedback and external focus feedback designs. ${ }^{9}{ }^{12}$ In addition, the skill of maintaining proper movements was found to be transferable to unlearned tasks, such as squatting and stair descent. ${ }^{12}$ The recent literature supports the role of corticomotor excitability in hip kinematics during weight-bearing activities in healthy individuals. ${ }^{13}$ These findings highlight the needs for examining the role of neurophysiological measures of the brain and spinal cord in individuals with PFP, as the mechanisms underlying altered neuromuscular control in this population remain to be poorly understood.

At the cortical level, the motor cortex plays a critical role in motor output, and altered motor cortex structure and function underlie movement dysfunction in individuals with PFP. Motor evoked potentials of quadriceps muscles in response to transcranial magnetic stimulations revealed altered corticomotor control in individuals with chronic PFP compared with asymptomatic individuals. ${ }^{14}$ Furthermore, persistent PFP has been reported to induce reorganisation of the primary motor cortex, with shifts in motor representations of all three quadriceps muscles, increased overlap of motor cortex representations and reduced volume, compared with asymptomatic individuals. ${ }^{15}$ At the level of the spinal cord, the H-reflex is a commonly used electrophysiological test for quantifying the excitatory behaviour of monosynaptic Ia afferent volleys in the spinal cord circuitry. This assessment of the Ia afferent-motoneuronal pathway is used for investigating the role and transmission of the spinal circuitry underlying motor control and its adaptations in movement disorders, lesions or training. ${ }^{16-19}$ Women with chronic PFP had significantly lower H-reflex amplitudes in the vastus medialis muscle and lower patellar tendon reflexes compared with pain-free individuals. Furthermore, the altered H-reflex amplitudes were strongly associated with pain levels, where women with PFP who had larger amplitudes of H-reflexes in the vastus medialis muscle had lower pain. ${ }^{20-22}$ Understanding the pathological neurophysiology underlying PFP is thus important for the future design of rehabilitation protocols targeting neural control underlying movement dysfunction in the population.

\section{OBJECTIVES}

The objective of this study is to evaluate the evidence for altered cortical and spinal cord functions in individuals with PFP.

\begin{tabular}{ll}
\hline Table 1 Search themes and search terms \\
\hline Search theme & Search terms \\
\hline Patellofemoral pain & 'patellofemoral pain' \\
& OR 'patellofemoral pain syndrome' \\
& OR 'patellofemoral syndrome' \\
& OR 'anterior knee pain' \\
& OR 'anterior knee pain syndrome' \\
& 'cortical reorganization' \\
& OR 'corticospinal excitability' \\
& OR 'transcranial magnetic stimulation' \\
& OR 'motor evoked potential' \\
& OR 'mapping' \\
& OR 'magnetic resonance imaging' \\
& OR 'functional magnetic resonance \\
imaging' & 'spinal excitability' \\
& OR 'H-reflex' \\
OR 'Hoffman reflex' & OR 'spinal reflex' \\
OR 'stretch reflex'
\end{tabular}

\section{METHODS}

\section{Protocol}

The protocol for conducting the review was prepared using the Preferred Reporting Items for Systematic Review and Meta-Analysis Protocols guidelines. ${ }^{23}$ In addition, this systematic review protocol has been registered with the International Prospective Register of Systematic Reviews.

\section{Search strategy}

We will conduct the literature search using the following databases: PubMed (MEDLINE), EMBASE and Web of Science. Search strings and Medical Subject Headings keywords related to the theme of PFP and non-invasive assessments of brain and spinal cord functions will be used (table 1). All search themes will be combined using the Boolean operators 'AND' and 'OR'. In addition, reviewers will manually screen the reference list of each article yielded from the search for additional articles. Methods for conducting this systematic review were developed using the Guidelines for Meta-Analysis and Systematic Reviews of Observational Studies. ${ }^{24}$

\section{Eligibility criteria}

The eligibility criteria for this study are shown in table 2 .

\section{Types of studies}

Peer-reviewed studies from cross-sectional, prospective, longitudinal, case-control and randomised control trial designs that examine the neurophysiological measures of cortical and spinal cord functions in persons with PFP will be included. Cross-sectional studies will be included if they meet the eligibility criteria (table 2). Case-control and randomised control trials will be included if the baseline data provide relevant information to the objective of this review. Prospective and longitudinal studies will be included if relevant data are available in individuals who develop PFP during the course of the study. 
Table 2 Inclusion and exclusion criteria for papers

\begin{tabular}{|c|c|}
\hline Inclusion criteria & Exclusion criteria \\
\hline $\begin{array}{l}\text { 1. Peer-reviewed, full-text } \\
\text { studies published in English } \\
\text { prior to } 1 \text { February } 2021 \\
\text { 2. Quantitative studies } \\
\text { using cross-sectional, } \\
\text { prospective, longitudinal, } \\
\text { case-control or randomised } \\
\text { control trial designs } \\
\text { 3. Participants are individuals } \\
\text { aged between } 18 \text { and } 45 \\
\text { years who have had PFP for } \\
\text { at least } 3 \text { months } \\
\text { 4. Studies including data } \\
\text { from a control group of } \\
\text { asymptomatic individuals } \\
\text { 5. Studies that investigate } \\
\text { the functions of the brain } \\
\text { and spinal cord using the } \\
\text { following assessments: } \\
\text { transcranial magnetic } \\
\text { stimulation, magnetic } \\
\text { resonance imaging } \\
\text { (including structural and } \\
\text { functional) and peripheral } \\
\text { nerve stimulation to assess } \\
\text { spinal circuit function } \\
\text { (including H-reflex gain, } \\
\text { recruitment curve slopes, } \\
\text { amplitudes) }\end{array}$ & $\begin{array}{l}\text { 1. Studies not published in } \\
\text { English } \\
\text { 2. Commentary, review or } \\
\text { editorial/opinion papers } \\
\text { 3. Theses, dissertation or } \\
\text { conference proceedings } \\
\text { 4. Participants younger } \\
\text { than } 18 \text { years of age } \\
\text { 5. Participants older than } \\
45 \text { years of age } \\
\text { 6. Participants report PFP } \\
\text { less than } 3 \text { months } \\
\text { 7. Studies that do not } \\
\text { include a control group } \\
\text { of individuals with no } \\
\text { PFP or studies that } \\
\text { use the asymptomatic } \\
\text { limb as the control for } \\
\text { comparisons }\end{array}$ \\
\hline
\end{tabular}

PFP, patellofemoral pain.

\section{Types of participants}

Participants are adults (aged bewteen 18 and 45 years $^{25}$ ) who have PFP. PFP is a common, chronic musculoskeletal condition, presenting as pain around or behind the patella during patellofemoral joint loading activities (eg, squatting, stair ambulation and running). ${ }^{34}$ Pain experienced by the participants should be greater than 3 months in duration, which is the chronic phase of pain. ${ }^{26}$ No restrictions will be placed on the sex. Studies with participants younger than 18 years will be excluded to avoid bias from including immature central nervous system function in paediatric/adolescent populations. Studies with participants older than 45 years will also be excluded to avoid the confounding findings related to patellofemoral joint osteoarthritis. Studies that do not include a control group of individuals with no PFP or studies that use the asymptomatic limb as the control for comparisons will also be excluded.

\section{Types of outcome measures}

Measures of neurophysiological changes should be reported in the eligible studies. These measures include cortical excitability as measured by transcranial magnetic stimulation, magnetic resonance imaging (including structural and functional) and peripheral nerve stimulation for assessment of spinal circuit function (ie, H-reflex gain and/or recruitment curve slopes and/or amplitudes elicited via nerve stimulations). The units of variables associated with H-reflex assessments are likely to vary and may include, but not limited to, millivolts, volts or ratios.

\section{Data management}

Two reviewers will evaluate the titles and abstracts of all studies yielded by the search with the inclusion and exclusion criteria, independently. Full texts of all eligible articles will be obtained and organised using EndNote X9 (Clarivate Analytics, Massachusetts) software. Duplicate studies will be removed. Should there be uncertainty or disagreement about the eligibility of a study between the two reviewers, we will consult an additional reviewer to reach a consensus.

\section{Data extraction}

The main data to be analysed within the abstract include descriptive information regarding the study (study design) and neurophysiological assessments that examine the functions of the brain and spinal cord using transcranial magnetic stimulation,magnetic resonance imaging (including structural and functional) and peripheral nerve stimulation for assessing spinal circuit function (H-reflex assessments).

\section{Risk of bias and quality assessment}

Two researchers will perform the assessment of risk of bias and quality independently. In the case of any disagreement, we will consult an additional reviewer. Eligible studies will be assessed by two investigators independently for methodological quality using National Institutes of Health's Quality Assessment Tool for Observational Cohort and Cross-Sectional Studies. ${ }^{27}$ This 14 -item assessment tool included 'yes', 'no', 'not applicable' and 'not reported' as possible responses. A score of 0 was given for 'no' and 1 for 'yes'. The methodological quality for each study will be categorised as good, fair or poor. ${ }^{27}$

\section{Strategy for data synthesis}

Effect size and 95\% CI will be calculated. We will use Cohen's d for analysis of effect size estimates, with $\mathrm{d}=0.2$ representing small effect size, $\mathrm{d}=0.5$ representing medium effect size and $d=0.8$ representing large effect size. ${ }^{28}$ Quantitative data will be presented as effect estimates. Statistical heterogeneity will be assessed using the $\mathrm{I}^{2}$ statistics, where $\mathrm{I}^{2}<25 \%$ represents low heterogeneity, $\mathrm{I}^{2}$ of $25 \%-50 \%$ represents medium heterogeneity and $\mathrm{I}^{2}>50 \%$ represents high heterogeneity. ${ }^{29}$ Statistical significance will be set at $p<0.05$. Narrative synthesis will be taken if a meta-analysis is not possible.

\section{Patient and public involvement}

The development of the research question and outcome measures was informed by the fact that patients with PFP often exhibit movement deficits, likely from changes in their neurophysiological function of the central nervous 
system. In this systematic review, we aim to examine the cortical and spinal cord functions in adult patients with $\mathrm{PFP}$, as PFP is a common disorder in this population. We plan to submit our findings to a peer-reviewed journal in the field of rehabilitation medicine, sports/orthopaedic medicine or neurology. As such, in addition to obtaining the research findings on their own, patients with PFP may receive the information from the healthcare professionals (eg, sports/orthopaedic physicians, physical therapists and athletic trainers).

\section{DISCUSSION}

This study will play an important role in evidence-based practice as it provides rich, in-depth understanding of the cortical and spinal cord functions in individuals with PFP through a thorough review and appraisal process. The analyses from the review findings will provide insight into the neurophysiological mechanisms underlying patellofemoral joint dysfunction, which in turn may be useful in improving movement patterns and symptoms in individuals with PFP. Efforts will also be made to conduct a meta-analysis; however, whether a synthesis of data can be made will depend on the variation between papers.

In addition to thorough analyses of findings in this topic, the strength and quality of the literature will be carefully examined. This information will guide future research in this field to better provide high-quality design and evidence in understanding the neurophysiological functions in individuals with PFP. We anticipated that the findings of this review will be of interest to various healthcare professionals, such as sports/orthopaedic physicians, physical therapists and athletic trainers, as well as to individuals who experience PFP.

\section{Ethics and dissemination}

This review meets the criteria for waiver of ethical approval, as defined by the Institutional Review Board at the University of Nevada, Las Vegas. The results of this study will be published in a peer-reviewed journal in the field of rehabilitation medicine, sports/orthopaedic medicine or neurology, regardless of the findings.

Contributors JNL and KH conceived and designed the study. JNL, SB, AM, KM, DN and $\mathrm{KH}$ developed the search strategies and analysis of the study. JNL, SB, AM, KM $\mathrm{DN}$ and $\mathrm{KH}$ were involved in writing and editing of the study protocol. All authors approved the final manuscript.

Funding This work was supported by the Student Opportunity Research Grant (SORG) from Department of Physical Therapy, University of Nevada, Las Vegas.

Disclaimer The funders had no role in study design, decision to publish or preparation of manuscript.

\section{Competing interests None declared.}

Patient and public involvement Patients and/or the public were involved in the design, or conduct, or reporting, or dissemination plans of this research. Refer to the Methods section for further details.

Patient consent for publication Not required.

Provenance and peer review Not commissioned; externally peer reviewed.

Open access This is an open access article distributed in accordance with the Creative Commons Attribution Non Commercial (CC BY-NC 4.0) license, which permits others to distribute, remix, adapt, build upon this work non-commercially, and license their derivative works on different terms, provided the original work is properly cited, appropriate credit is given, any changes made indicated, and the use is non-commercial. See: http://creativecommons.org/licenses/by-nc/4.0/.

\section{ORCID iDs}

Jing Nong Liang http://orcid.org/0000-0003-2497-1496

Kai-Yu Ho http://orcid.org/0000-0002-4871-1800

\section{REFERENCES}

1 Smith BE, Selfe J, Thacker D, et al. Incidence and prevalence of patellofemoral pain: a systematic review and meta-analysis. PLoS One 2018;13:e0190892.

2 Roush JR, Curtis Bay R. Prevalence of anterior knee pain in 18-35 year-old females. Int J Sports Phys Ther 2012;7:396-401.

3 Crossley KM, Stefanik JJ, Selfe J, et al. 2016 Patellofemoral pain consensus statement from the 4th International Patellofemoral pain research retreat, Manchester. Part 1: terminology, definitions, clinical examination, natural history, patellofemoral osteoarthritis and patientreported outcome measures. Br J Sports Med 2016;50:839-43.

4 Collins NJ, Barton CJ, van Middelkoop M, et al. 2018 consensus statement on exercise therapy and physical interventions (orthoses, taping and manual therapy) to treat patellofemoral pain: recommendations from the 5th International Patellofemoral pain research retreat, gold Coast, Australia, 2017. Br J Sports Med 2018;52:1170-8

5 Powers CM, Witvrouw E, Davis IS, et al. Evidence-based framework for a pathomechanical model of patellofemoral pain: 2017 patellofemoral pain consensus statement from the 4th International Patellofemoral pain research retreat, Manchester, UK: Part 3. Br J Sports Med 2017;51:1713-23.

6 Scholtes SA, Salsich GB. A dynamic valgus index that combines hip and knee angles: assessment of utility in females with patellofemoral pain. Int J Sports Phys Ther 2017;12:333-40.

7 Ferrari D, Briani RV, de Oliveira Silva D, et al. Higher pain level and lower functional capacity are associated with the number of altered kinematics in women with patellofemoral pain. Gait Posture 2018;60:268-72.

8 Willy RW, Hoglund LT, Barton CJ, et al. Patellofemoral pain. J Orthop Sports Phys Ther 2019;49:CPG1-95.

9 Davis IS, Tenforde AS, Neal BS, et al. Gait retraining as an intervention for Patellofemoral pain. Curr Rev Musculoskelet Med 2020;13:103-14.

10 Rabelo NDDA, Lucareli PRG. Do hip muscle weakness and dynamic knee valgus matter for the clinical evaluation and decision-making process in patients with patellofemoral pain? Braz J Phys Ther 2018;22:105-9

11 Neal BS, Lack SD, Lankhorst NE, et al. Risk factors for patellofemoral pain: a systematic review and meta-analysis. $\mathrm{Br} J$ Sports Med 2019;53:270-81.

12 Willy RW, Scholz JP, Davis IS. Mirror gait retraining for the treatment of patellofemoral pain in female runners. Clin Biomech 2012;27:1045-51.

13 Shih Y, Fisher BE, Smith JA, et al. Corticomotor excitability of gluteus maximus is associated with hip biomechanics during a Single-Leg Drop-Jump. J Mot Behav 2021;53:40-6.

14 On AY, Uludağ B, Tașkiran E, et al. Differential corticomotor control of a muscle adjacent to a painful joint. Neurorehabil Neural Repair 2004;18:127-33.

15 Te M, Baptista AF, Chipchase LS, et al. Primary motor cortex organization is altered in persistent Patellofemoral pain. Pain Med 2017;18:2224-34.

16 Liang JN, Brown DA. Impaired H-reflex gain during postural loaded locomotion in individuals post-stroke. PLoS One 2015;10:e0144007.

17 Liang JN, Lee Y-J, Akoopie E, et al. Impaired H-reflex adaptations following slope walking in individuals with post-stroke hemiparesis. Front Physiol 2019;10:1232.

18 Pierrot-Deseilligny E, Burke D. The circuitry of the human spinal cord: spinal and corticospinal mechanisms of movement. Cambridge: Cambridge University Press, 2012.

19 Schieppati M. The Hoffmann reflex: a means of assessing spinal reflex excitability and its descending control in man. Prog Neurobiol 1987;28:345-76.

20 de Oliveira Silva D, Magalhães FH, Faria NC, et al. Vastus medialis Hoffmann reflex excitability is associated with pain level, selfreported function, and chronicity in women with Patellofemoral pain. Arch Phys Med Rehabil 2017;98:114-9. 
21 de Oliveira Silva D, Magalhães FH, Faria NC, et al. Lower amplitude of the Hoffmann reflex in women with Patellofemoral pain: thinking beyond proximal, local, and distal factors. Arch Phys Med Rehabil 2016:97:1115-20.

22 Pazzinatto MF, de Oliveira Silva D, Ferreira AS, et al. Patellar tendon reflex and vastus medialis Hoffmann reflex are down regulated and correlated in women with Patellofemoral pain. Arch Phys Med Rehabil 2019;100:514-9.

23 Shamseer L, Moher D, Clarke M, et al. Preferred reporting items for systematic review and meta-analysis protocols (PRISMA-P) 2015: elaboration and explanation. BMJ 2015;349:g7647.

24 Stroup DF, Berlin JA, Morton SC, et al. Meta-analysis of observational studies in epidemiology: a proposal for reporting. Meta-analysis of observational studies in epidemiology (moose) group. JAMA 2000;283:2008-12.
25 Ho K-Y, Chen Y-J, Farrokhi S, et al. Selective atrophy of the vastus medialis: does it exist in women with nontraumatic Patellofemoral pain? Am J Sports Med 2021;49:700-5.

26 Ho K-Y, Hu HH, Colletti PM, et al. Running-induced patellofemoral pain fluctuates with changes in patella water content. Eur J Sport Sci 2014;14:628-34.

27 Study quality assessment tools. Available: https://www.nhlbi.nih.gov/ health-topics/study-quality-assessment-tools

28 Cohen J. Statistical power analysis for the behavioral sciences. 2nd ed. Routledge, 1988.

29 Melsen WG, Bootsma MCJ, Rovers MM, et al. The effects of clinical and statistical heterogeneity on the predictive values of results from meta-analyses. Clin Microbiol Infect 2014;20:123-9. 